\title{
Penetration of plasma into the wafer-focus ring gap in capacitively coupled plasmas
}

\author{
Natalia Y. Babaeva ${ }^{\text {a) }}$ and Mark J. Kushner ${ }^{\text {b) }}$ \\ Iowa State University, Department of Electrical and Computer Engineering, 104 Marston Hall, Ames, Iowa \\ 50011
}

(Received 17 February 2006; accepted 27 March 2007; published online 8 June 2007)

\begin{abstract}
In plasma etching equipment for microelectronics fabrication, there is an engineered gap between the edge of the wafer and wafer terminating structures, such as focus rings. The intended purpose of these structures is to make the reactant fluxes uniform to the edge of the wafer and so prevent a larger than desired edge exclusion where useful products cannot be obtained. The wafer-focus ring gap (typically $<1 \mathrm{~mm}$ ) is a mechanical requirement to allow for the motion of the wafer onto and off of the substrate. Plasma generated species can penetrate into this gap and under the beveled edge of the wafer, depositing films and possibly creating particles which produce defects. In this paper, we report on a computational investigation of capacitively coupled plasma reactors with a wafer-focus ring gap. The penetration of plasma generated species (i.e., ions and radicals) into the wafer-focus ring gap is discussed. We found that the penetration of plasma into the gap and under the wafer bevel increases as the size of the gap approaches and exceeds the Debye length in the vicinity of the gap. Deposition of, for example, polymer by neutral species inside the gap and under the wafer is less sensitive to the size of the gap due the inability of ions, which might otherwise sputter the film, to penetrate into the gap. () 2007 American Institute of Physics.
\end{abstract}

[DOI: $10.1063 / 1.2736333$ ]

\section{INTRODUCTION}

The method of terminating the edge of the wafer in plasma processing of microelectronic devices has important implications. When transitioning across the edge of the wafer to nonwafer surfaces, there are changes in the electrical, material, and geometrical properties of the substrate. These affects can translate into a change in the plasma produced fluxes of radicals and ions to the edge of the wafer, usually toward being less optimum. The end result is that the outer few $\mathrm{mm}$ of radii of wafers are often not usable for producing devices. As the diameter of wafers increases, the number of devices that are not able to be fabricated in these edge regions also increases, now resulting in 5\%-7\% of the wafer not being productive in terms of producing devices.

A second edge effect is an inevitable gap between the wafer and the terminating substrate structure, often called the focus ring, as shown in Fig. 1. A gap between the wafer and the focus ring is needed for mechanical clearance to allow for placement and removal of the wafer within tolerances of the wafer handling system. The gap is a few tenths of a mm to a few $\mathrm{mm}$. The edge of the wafer is often beveled and rounded to eliminate sources of mechanical stress and to maximize ease of wafer handling.

Penetration of plasma produced species (electrons, ions, and neutral radicals) into the wafer-focus ring gap can have a number of undesirable consequences. Films can be deposited on surfaces in the wafer-focus ring gap, on the bevel, and even on the back-side of the wafer. These films may be, for

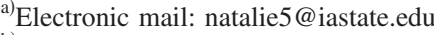

b) Author to whom correspondence should be addressed. Electronic mail: mjk@iastate.edu
}

example, polymers as produced in fluorocarbon gas mixtures for dielectric etch, the desired product of deposition chemistries or redeposition of etch products. The volume within the wafer-focus ring gap may also be a source of contamination by providing a site for particle formation. There is some evidence that the beveled edge of the wafer may be an attractor for contamination, with metals and particles preferentially adhering there, perhaps the result of some electrical focusing on the edge of the bevel. ${ }^{1}$

The sheath thickness in capacitively coupled plasma tools can be greater than or commensurate to the wafer-focus

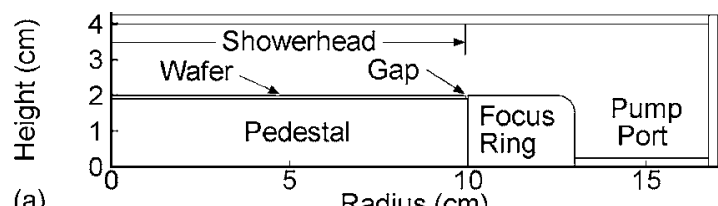

(a)

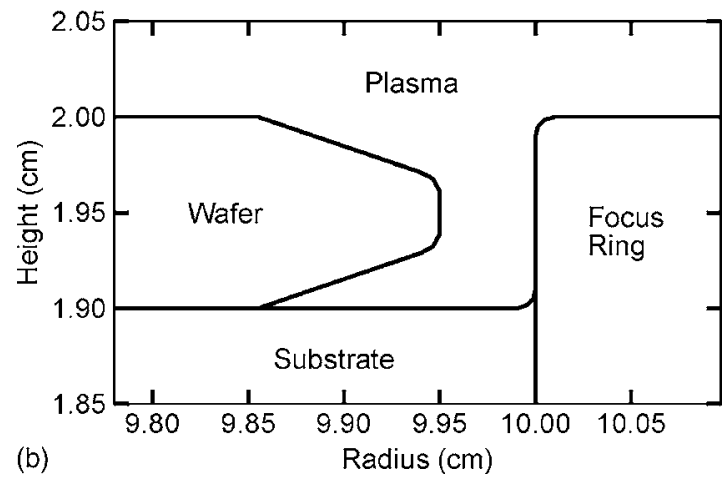

FIG. 1. Schematics of the geometry used in this investigation. (a) Full geometry showing the location of the wafer-focus ring gap and (b) a close up of the wafer-focus ring gap. 
TABLE I. Reduced reaction mechanism for $\mathrm{Ar} / \mathrm{CF}_{4}$ plasmas.

\begin{tabular}{llll}
\hline \hline & & Species & \\
\hline $\mathrm{Ar}$ & $\mathrm{CF}_{4}$ & $\mathrm{CF}_{3}^{+}$ & \\
$\mathrm{Ar}^{*}(4 s)$ & $\mathrm{CF}_{3}$ & $\mathrm{CF}_{3}^{-}$ & \\
$\mathrm{Ar}^{* *}(4 p)$ & $\mathrm{CF}_{2}$ & $\mathrm{~F}^{-}$ & \\
$\mathrm{Ar}^{+}$ & $\mathrm{CF}$ & $e$ & \\
& $\mathrm{~F} 2$ & & \\
& $\mathrm{~F}$ & & \\
& Reactions $^{\mathrm{a}}$ & Rate coefficient & \\
& & & Reference
\end{tabular}

Electron Impact:

$e+\mathrm{Ar} \rightarrow \mathrm{Ar}^{*}+e$

$e+\mathrm{Ar} \rightarrow \mathrm{Ar}^{* *}+e$

$e+\mathrm{Ar} \rightarrow \mathrm{Ar}^{+}+e+e$

$e+\mathrm{Ar}^{*} \rightarrow \mathrm{Ar}^{+}+e+e$

$e+\mathrm{Ar} * \rightarrow \mathrm{Ar}+e$

$e+\mathrm{Ar}^{*} \rightarrow \mathrm{Ar}^{* *}+e$

$e+\mathrm{Ar}^{* *} \rightarrow \mathrm{Ar}+e$

$e+\mathrm{Ar}^{* *} \rightarrow \mathrm{Ar}^{+}+e$

$e+\mathrm{Ar}^{* *} \rightarrow \mathrm{Ar}^{*}+e$

$e+\mathrm{CF}_{4} \rightarrow \mathrm{CF}_{3}+\mathrm{F}^{-}$

$e+\mathrm{CF}_{4} \rightarrow \mathrm{CF}_{3}^{-}+\mathrm{F}$

$e+\mathrm{CF}_{4} \rightarrow \mathrm{CF}_{3}+\mathrm{F}+e$

$e+\mathrm{CF}_{4} \rightarrow \mathrm{CF}_{3}^{+}+\mathrm{F}+e+e$

$e+\mathrm{CF}_{4} \rightarrow \mathrm{CF}_{2}+\mathrm{F}+\mathrm{F}+e$

$e+\mathrm{CF}_{4} \rightarrow \mathrm{CF}_{3}^{+}+\mathrm{F}^{-}+e$

$e+\mathrm{CF}_{4} \rightarrow \mathrm{CF}+\mathrm{F}+\mathrm{F}_{2}+e$

$e+\mathrm{CF}_{3} \rightarrow \mathrm{CF}_{2}+\mathrm{F}+e$

$e+\mathrm{CF}_{3} \rightarrow \mathrm{CF}_{3}^{+}+e+e$

$e+\mathrm{CF}_{3} \rightarrow \mathrm{CF}_{2}+\mathrm{F}^{-}$

$e+\mathrm{CF}_{2} \rightarrow \mathrm{CF}+\mathrm{F}^{-}$

$e+\mathrm{CF}_{2} \rightarrow \mathrm{CF}+\mathrm{F}+e$

$e+\mathrm{F}_{2} \rightarrow \mathrm{F}^{-}+\mathrm{F}$

Neutral heavy particle reactions:

$\mathrm{Ar}^{*}+\mathrm{Ar}^{*} \rightarrow \mathrm{Ar}^{+}+\mathrm{Ar}+e$

$\mathrm{Ar}^{* *}+\mathrm{Ar}^{* *} \rightarrow \mathrm{Ar}^{+}+\mathrm{Ar}+e$

$\mathrm{Ar}^{* * *}+\mathrm{Ar}^{*} \rightarrow \mathrm{Ar}^{+}+\mathrm{Ar}+e$

$\mathrm{Ar}^{* *} \rightarrow \mathrm{Ar}^{*}$

$\mathrm{F}+\mathrm{CF}_{3} \rightarrow \mathrm{CF}_{4}$

$\mathrm{F}+\mathrm{CF}_{2} \rightarrow \mathrm{CF}_{3}$

$\mathrm{F}+\mathrm{CF} \rightarrow \mathrm{CF}_{2}$

$\mathrm{F}_{2}+\mathrm{CF}_{2} \rightarrow \mathrm{CF}_{3}+\mathrm{F}$

$\mathrm{F}_{2}+\mathrm{CF}_{3} \rightarrow \mathrm{CF}_{4}+\mathrm{F}$

$\mathrm{Ar}^{*}+\mathrm{CF}_{4} \rightarrow \mathrm{CF}_{2}+\mathrm{F}_{2}+\mathrm{Ar}$

$\mathrm{Ar}^{*}+\mathrm{CF}_{3} \rightarrow \mathrm{CF}_{2}+\mathrm{F}+\mathrm{Ar}$

$\mathrm{Ar}^{*}+\mathrm{CF}_{2} \rightarrow \mathrm{CF}+\mathrm{F}+\mathrm{Ar}$

Ion-neutral particle reactions:

$\mathrm{Ar}^{+}+\mathrm{Ar} \rightarrow \mathrm{Ar}+\mathrm{Ar}^{+}$

$\mathrm{CF}_{3}^{+}+\mathrm{CF}_{3} \rightarrow \mathrm{CF}_{3}+\mathrm{CF}_{3}^{+}$

$\mathrm{CF}_{3}^{-}+\mathrm{F} \rightarrow \mathrm{CF}_{3}+\mathrm{F}^{-}$

$\mathrm{F}^{-}+\mathrm{CF}_{3} \rightarrow \mathrm{CF}_{4}+e$

$\mathrm{F}^{-}+\mathrm{CF}_{2} \rightarrow \mathrm{CF}_{3}+e$

$\mathrm{F}^{-}+\mathrm{CF} \rightarrow \mathrm{CF}_{2}+e$

$\mathrm{F}^{-}+\mathrm{F} \rightarrow \mathrm{F}_{2}+e$

$\mathrm{Ar}^{+}+\mathrm{CF}_{4} \rightarrow \mathrm{CF}_{3}^{+}+\mathrm{Ar}+\mathrm{F}$

$\mathrm{Ar}^{+}+\mathrm{CF}_{3} \rightarrow \mathrm{CF}_{3}^{+}+\mathrm{Ar}$

Electron-ion and ion-ion reactions:

$\mathrm{e}+\mathrm{Ar}^{+} \rightarrow \mathrm{Ar}^{* *}$

$e+\mathrm{CF}_{3}^{+} \rightarrow \mathrm{CF}_{2}+\mathrm{F}$

$\mathrm{CF}_{3}^{-}+\mathrm{CF}_{3}^{+} \rightarrow \mathrm{CF}_{3}+\mathrm{CF}_{3}$

$\mathrm{F}^{-}+\mathrm{CF}_{3}^{+} \rightarrow \mathrm{CF}_{2}+\mathrm{F}_{2}$

$\mathrm{F}^{-}+\mathrm{CF}_{3}^{+} \rightarrow \mathrm{CF}_{2}+\mathrm{F}+\mathrm{F}$

$\mathrm{F}^{-}+\mathrm{CF}_{3}^{+} \rightarrow \mathrm{F}+\mathrm{CF}_{3}$

$\mathrm{CF}_{3}^{-}+\mathrm{Ar}^{+} \rightarrow \mathrm{CF}_{3}+\mathrm{Ar}$
Rate coefficient ${ }^{\mathrm{b}}$

Reference

11

12

10

13

10

14

13

15

15

15

$15^{\mathrm{d}}$

15

15

15

$15^{\mathrm{f}}$

16

$15^{\mathrm{f}}$

$15^{\mathrm{f}}$

$15^{\mathrm{f}}$

17

$1.0 \times 10^{-9}$

$1.0 \times 10^{-9}$

$1.0 \times 10^{-9}$

$2 \times 10^{6} \mathrm{~s}^{-1}$

$2.0 \times 10^{-11}$

$1.8 \times 10^{-11}$

$9.96 \times 10^{-11}$

$8.3 \times 10^{-14}$

$1.88 \times 10^{-14}$

$4.0 \times 10^{-11}$

$4.0 \times 10^{-11}$

$4.0 \times 10^{-11}$

$4.6 \times 10^{-10}$

$1.0 \times 10^{-9}$

$5.0 \times 10^{-8}$

$4.0 \times 10^{-10}$

$3.0 \times 10^{-10}$

$2.0 \times 10^{-10}$

$1.0 \times 10^{-10}$

$4.8 \times 10^{-10}$

$7.0 \times 10^{-10}$

$4.0 \times 10^{-13} T_{e}^{-0.5}$

$3.0 \times 10^{-8} T^{-0.5}$

$3.0 \times 10^{-7}$

$8.7 \times 10^{-8}$

$3.0 \times 10^{-7}(T / 300)^{-0.5}$

$3.0 \times 10^{-7}$

$3.0 \times 10^{-7}$
TABLE I. (Continued.)

\begin{tabular}{lcc}
\hline \hline & \multicolumn{2}{c}{ Species } \\
\hline $\mathrm{F}^{-}+\mathrm{Ar}^{+} \rightarrow \mathrm{F}+\mathrm{Ar}$ & $5.0 \times 10^{-7}$ & 30 \\
\hline
\end{tabular}

$\overline{{ }^{a} \text { Only reactions directly affecting species densities are shown here. Addi- }}$ tional electron impact collisions such as momentum transfer and excitation of vibrational and electronic states are included in the solution of Boltzmann's equation for the electron energy distribution to account for the transport and energy losses of electrons.

${ }^{b}$ Rate coefficients have units of $\mathrm{cm}^{3} \mathrm{~s}^{-1}$ unless notes otherwise.

${ }^{\mathrm{c}}$ Computed using the electron energy distribution and electron impact cross section from cited reference.

${ }^{\mathrm{d}}$ We lumped together the four sublevels of $\operatorname{Ar}^{*}(4 s)$. This reaction takes into account the decay of the two radiative $\operatorname{Ar}^{*}(4 s)$ sublevels.

${ }^{\mathrm{e}}$ Estimated for a mildly trapped optical transition.

f Estimated by analogy to $\mathrm{CF}_{4}$.

${ }^{\mathrm{g}}$ Estimated.

ring gap dimensions. The shape of the sheath can, therefore, by influenced by the wafer-focus ring gap topology, dielectric constant, and conductivity of the wafer and focus ring. If the size of the sheath is commensurate to the size of the gap, the sheath may tend to wrap around the contour of the features (plasma molding) which can significantly modify the orientation of the electric field and ion trajectories. ${ }^{2,3}$ Plasma molding over steps, trenches, and holes, and the resulting distributions of ions and fast neutrals have been recently investigated. ${ }^{4,5}$ These studies are particularly relevant to the production of energetic neutral beam sources for etching in which ions are neutralized during their transit through high aspect ratio holes having dimensions commensurate with sheath thickness.

In this paper, we discuss results from a computational investigation of plasma penetration into the wafer-focus ring gap in capacitively coupled plasmas sustained in a mildly polymerizing $\mathrm{Ar} / \mathrm{CF}_{4}$ chemistry. The model used in this investigation is a two-dimensional (2D) plasma hydrodynamics simulation utilizing an unstructured mesh to resolve the small dimensions of the wafer-focus ring gap. Improvements were made to the model to capture the effects of ion inertia by solving the full momentum equations for ions, including momentum transfer between ions and neutrals. The consequences of the width of the wafer-focus ring gap and the height of the focus ring will be discussed in the context of plasma and radical penetration into the gap and under the wafer. In particular, the likelihood for polymer deposition into the gap will be discussed.

We found that there is a fairly sharp transition in the propensity for penetration of plasma into the gap as a function of width of the gap. This is correlated with the width of the sheath. As the width of the gap increases to the thickness of the sheath, plasma penetration significantly increases. The propensity for polymer deposition in the gap is also high, at least in relative terms. The ratio of ion flux to polymerizing radical flux decreases in the gap, particularly for small gaps. The result is that the removal of film by sputtering decreases, producing a net increase in effective sticking coefficient of the neutral species.

The model used in this investigation will be briefly described in Sec. II, followed by a discussion of the reaction 
a)

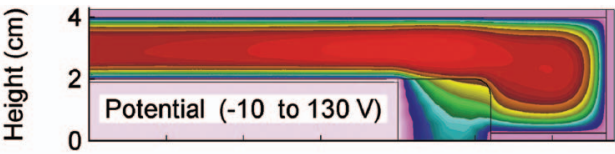

b)
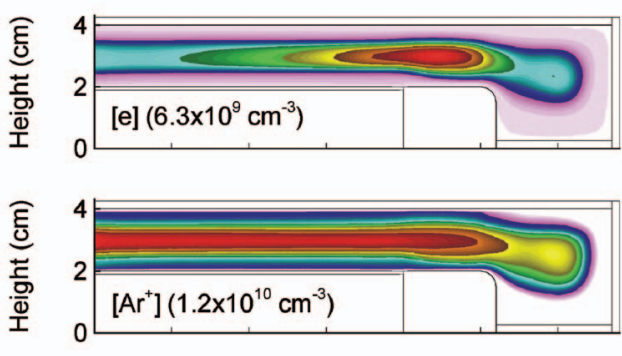

c)

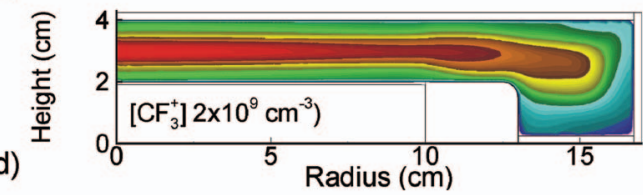

MIN

MAX

FIG. 2. (Color) Plasma properties averaged over the rf cycle for the base case conditions ( $\mathrm{Ar} / \mathrm{CF}_{4}=97 / 3,90 \mathrm{mTorr}, 300 \mathrm{~V}, 10 \mathrm{MHz}$ ). (a) Plasma potential, (b) electron density, (c) $\mathrm{Ar}^{+}$density, and (d) $\mathrm{CF}_{3}^{+}$density. The maximum value for each frame is shown. Although the electron density peaks near the edge of the wafer, the ion density is uniform across the wafer

mechanism in Sec. III. The results of our investigation are discussed in Sec. IV. Our concluding remarks are in Sec. V.

\section{DESCRIPTION OF THE MODEL}

The model used in this investigation, nonPDPSIM, is a multifluid hydrodynamics simulation in which transport equations for all charged and neutral species, Poisson's equation and a surface kinetics model are integrated as a function of time using unstructured meshes. ${ }^{6}$ The model will be briefly described, including improvements to algorithms to resolve ion momentum.

The fundamental equations solved in nonPDPSIM for charged particle transport are the continuity equation for species densities, Poisson's equation for the electric potential, and charge densities on and inside materials

$$
\begin{aligned}
& \frac{\partial N_{j}}{\partial t}=-\nabla \cdot \vec{\Gamma}_{j}+S_{j}, \\
& -\nabla(\varepsilon \nabla \Phi)=\sum_{j} q_{j} N_{j}+\rho_{m}, \\
& \frac{\partial \rho_{m}}{\partial t}=\left[\sum_{j} q_{j}\left(-\nabla \cdot \vec{\Gamma}_{j}\right)-\nabla \cdot \sigma_{m} \vec{E}\right]_{m},
\end{aligned}
$$

where $N_{j}$ is the density of species $j$ having flux $\vec{\Gamma}_{j}$, and source function $S_{j}$. $\Phi$ is the electrical potential, $E$ is the electric field, $\varepsilon$ is the permittivity, $\sigma_{m}$ is the material conductivity, and $\rho_{m}$ is the charge density in and on materials. The bracketed subscript in Eq. (3) indicates that the $\rho_{m}$ is only calculated on and in materials. The fluxes for electrons are given by the Scharfetter-Gummel formulation.

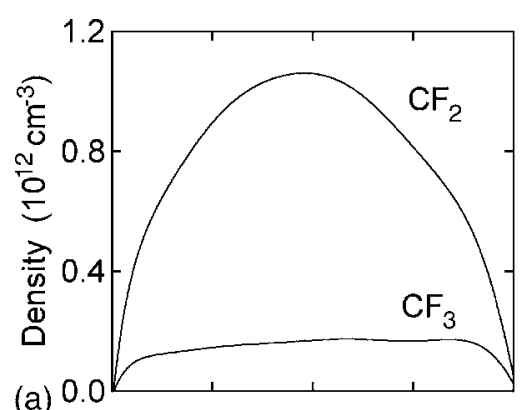

(a)

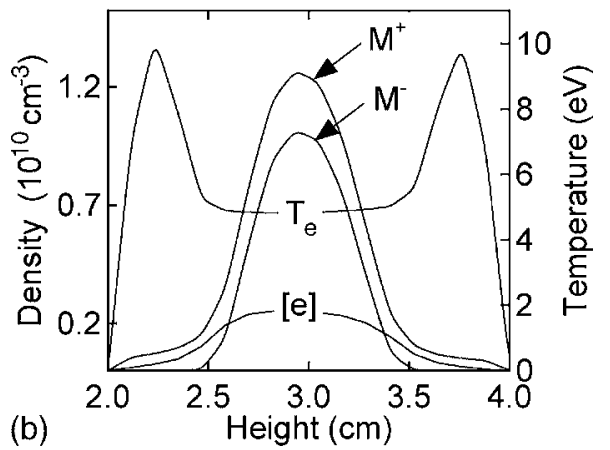

FIG. 3. Plasma properties as a function of height on the axis $(r=0)$ for the base case conditions. (a) $\mathrm{CF}_{2}$ and $\mathrm{CF}_{3}$ densities, and (b) sum of positive and negative ion densities, and electron density, and temperature.

As an improvement to the model, the flux of ion $j$ is obtained by solving the ion momentum equation

$$
\begin{aligned}
\frac{\partial \vec{\Gamma}_{j}}{\partial t}= & -\frac{1}{M_{j}} \nabla P_{j}-\nabla\left(N_{j} \vec{V}_{j} \vec{V}_{j}\right)+\frac{q_{j} N_{j} \vec{E}}{M_{j}} \\
& -\sum_{i} N_{j} \nu_{i j}\left(\vec{V}_{j}-\vec{V}_{i}\right),
\end{aligned}
$$

where $P_{j}=N_{j} k T_{j}$ is the ion partial pressure for ion temperature $T_{j}, M_{j}$ is the ion mass, $V_{j}$ is the velocity of species $j$, and $\nu_{i j}$ is the momentum transfer collision frequency between species $i$ and $j$. The terms in Eq. (4) account for changes in momentum due to the pressure gradients, convection, acceleration by the electric field, and momentum transfer between species. The summation is over both neutral and ion species, though the collisions with neutrals is the dominant term. We have ignored viscosity effects due to the low partial pressure of ions.

Two options for solving the system of equations with ion momentum can be used, fully implicit and semi-implicit. In the fully implicit method, Eqs. (1) and (4) are couched in terms of $\Delta M_{i k}=F_{i k}\left(\Delta t, M_{j l}\right)$ where $\Delta M_{i k}$ is the change in quantity $k$ (charged particle density, electric potential, material charge density, radial, and axial component of ion momentum flux) at mesh point $i$ during time step $\Delta t . F_{j k}$ is a function which expresses the dependence of $\Delta M_{i k}$ on $\Delta t$, other quantities being implicitly solved for at $i$ and other mesh points $j$. (Notations for the dependence of $\Delta M_{i k}$ on other quantities not being implicitly solved for in this time step have been suppressed.) Jacobian elements, $\partial F_{i k} / \partial F_{j l}$ are either analytically or numerically formulated. When numerically formulating the Jacobian elements, the $M_{j l}$ are perturbed by a small amount to determine the resulting change 

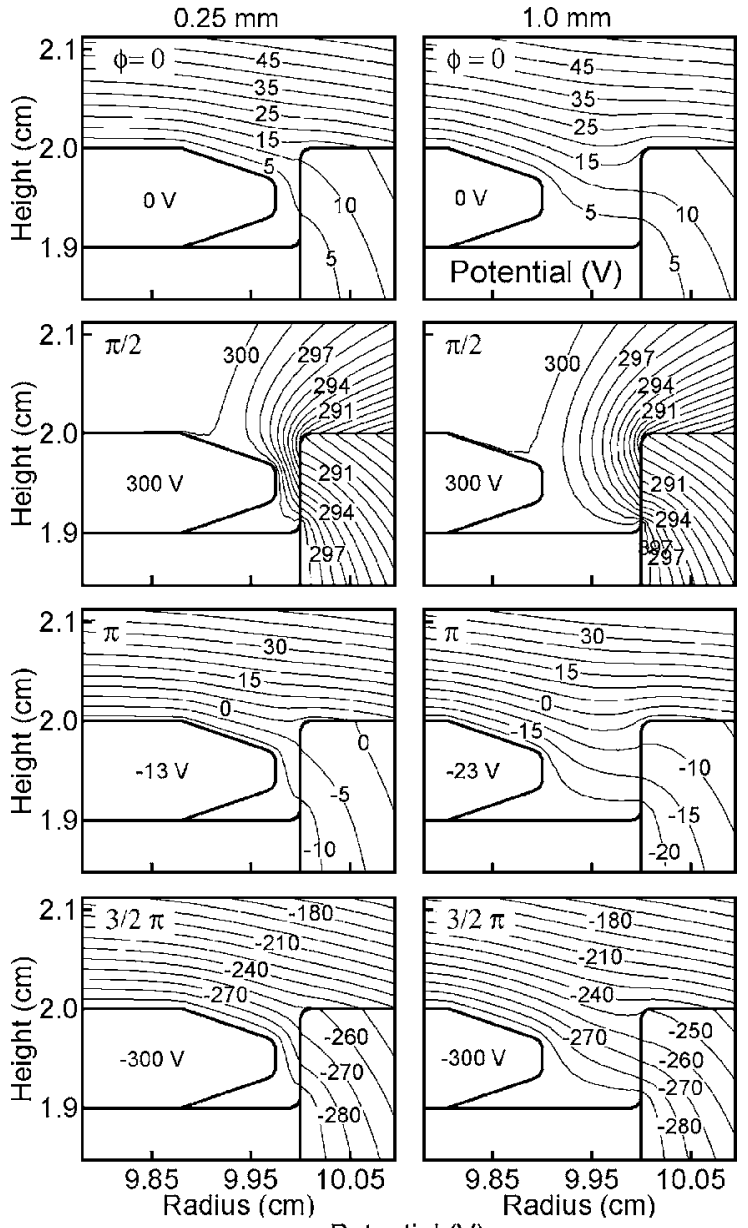

Potential $(V)$

FIG. 4. Plasma potential (volts) at different phases during the rf cycle for (left) a $0.25 \mathrm{~mm}$ gap and (right) a $1 \mathrm{~mm}$ gap for the base case conditions. The maximum voltage occurs at $\phi=\pi / 2$ and minimum voltage at $3 \pi / 2$.

in $M_{i k}$. This system of equations is expressed in matrix form and implicitly solved using a modified Newton's method. The second method is to exclude the equations for components of momentum from the matrix; and directly integrate the momentum equations after the implicit update of the charged particle densities and potential.

In a time slicing fashion, the bulk electron temperature and neutral particle densities are updated following the update of charge particle densities, ion fluxes, and electric potential. The equation solved for electron temperature is

$$
\frac{\partial\left(n_{e} \varepsilon\right)}{\partial t}=q \vec{\Gamma}_{e} \cdot \vec{E}-n_{e} \sum_{j} N_{j} k_{j} \Delta \varepsilon_{j}-\nabla \cdot\left(\frac{5}{2} \varepsilon \vec{\Gamma}_{e}-\lambda_{e} \nabla T_{e}\right),
$$

where the average electron energy $\varepsilon=\frac{3}{2} k T_{e}$ for electron temperature $T_{e}, \Gamma_{e}$ is the Scharfetter-Gummel form of the electron flux, and $\lambda_{e}$ is the electron thermal conductivity. The summation is over electron collisions with species having density $N_{j}$ and rate coefficient $k_{j}$ resulting in change in electron energy $\Delta \varepsilon_{j}$. The electron transport coefficients and rate coefficients for bulk electrons as a function of $T_{e}$ are obtained by solving Boltzmann's equation for the electron energy distribution. Transport of secondary electrons from the

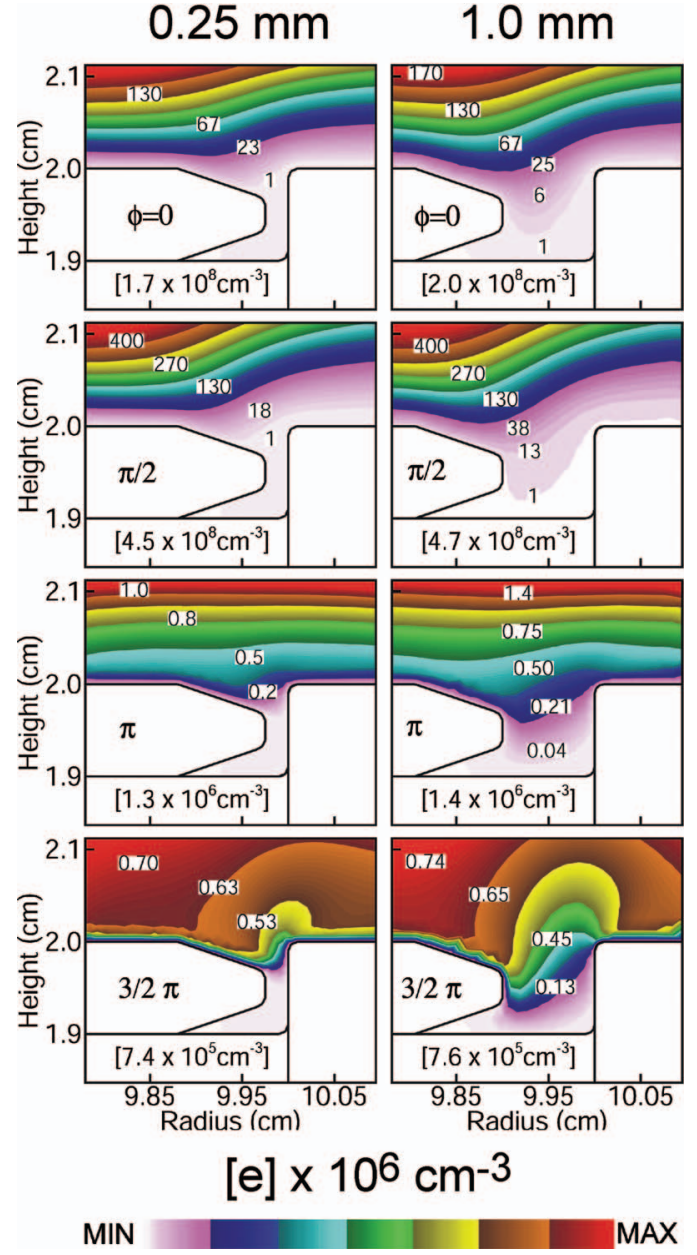

FIG. 5. (Color) Electron density at different phases during the rf cycle for (left) a $0.25 \mathrm{~mm}$ gap and (right) a $1 \mathrm{~mm}$ gap for the base case conditions. The maximum voltage occurs at $\phi=\pi / 2$ and the minimum voltage at $3 \pi / 2$. Contour labels are in units of $10^{6} \mathrm{~cm}^{-3}$. Each frame is separately normalized to its maximum value. Electron penetration into the gap is nominal for the smaller gap.

biased substrate is addressed using a Monte Carlo simulation.

The surface kinetics module consists of a surface site balance model solved at every point along the plasmasurface boundary. Although implemented in a different modeling platform, the methodology of the surface kinetics model, including enabling there to be multiple layer of polymers, is essentially the same as that described in Ref. 7.

Neutral transport is addressed by solving the NavierStokes equations for the bulk neutral fluid flow

$$
\frac{\partial \rho}{\partial t}=-\nabla \cdot(\rho \vec{V})+P,
$$

$$
\begin{aligned}
\frac{\partial(\rho \vec{V})}{\partial t}= & -\nabla p-\nabla \cdot(\rho \vec{V} \vec{V})-\nabla \cdot \overline{\bar{\tau}} \\
& +\sum_{j}\left[q_{j} \vec{E}+M_{j} \nu_{j}\left(\vec{V}_{j}-\vec{V}\right)\right] N_{j},
\end{aligned}
$$




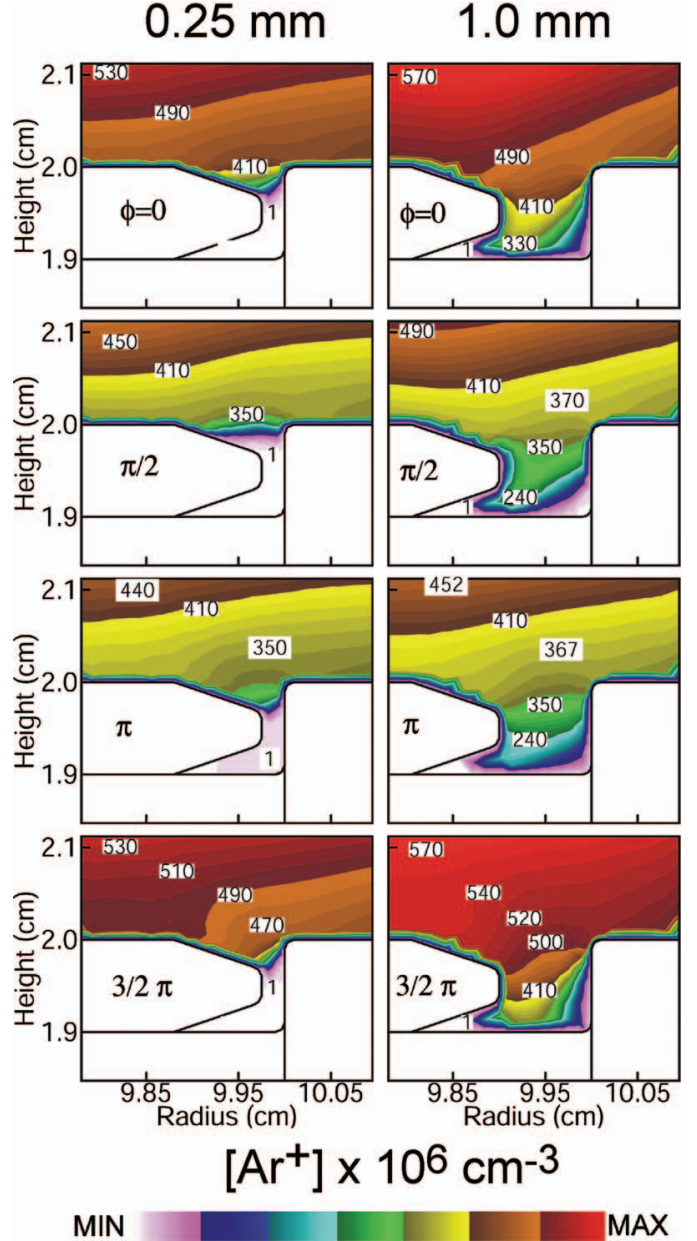

FIG. 6. (Color) $\mathrm{Ar}^{+}$density at different phases during the rf cycle for (left) a $0.25 \mathrm{~mm}$ gap and (right) a $1 \mathrm{~mm}$ gap for the base case conditions. The maximum voltage occurs at $\phi=\pi / 2$ and minimum voltage at $3 \pi / 2$. Contour labels are in units of $10^{6} \mathrm{~cm}^{-3}$. Ions penetrate into the larger gap throughout the rf cycle.

$$
\begin{aligned}
\frac{\partial\left(\rho c_{p} T\right)}{\partial t}= & -\nabla\left(-\kappa \nabla T+\rho \vec{V} c_{p} T\right) \\
& +\sum_{j} \overrightarrow{j_{j}} \cdot \vec{E}-\sum_{i} R_{i} \Delta H_{i}+p \nabla \cdot \vec{V},
\end{aligned}
$$

where $P$ represents the inlet and outlet flows (the inlet flow is specified while the output flow is adjusted to maintain a constant mass flux), $\rho$ is the total mass density, $V$ is the neutral fluid velocity, $p$ is the thermodynamic pressure, $\overline{\bar{\tau}}$ is the viscosity tensor, $c_{p}$ is the heat capacity, $\kappa$ is the species averaged thermal conductivity, and $M$ is molecular weight. The subscripts $j$ are for summations over charged species. The last term in Eq. (7) accounts for volume forces and momentum transfer from charged particles to neutrals. The contribution to the energy equation from Joule heating contains contributions from ions. $\Delta H_{i}$ is the change enthalpy due to reaction $i$ having a total rate of $R_{i}$. The reactions include Frank-Condon gas heating from electron-impact dissociation of molecules and elastic collisions with electrons as well as conventional chemical reactions.

The weakness to our approach is the large Knudsen number that may occur within the gap. For the particular

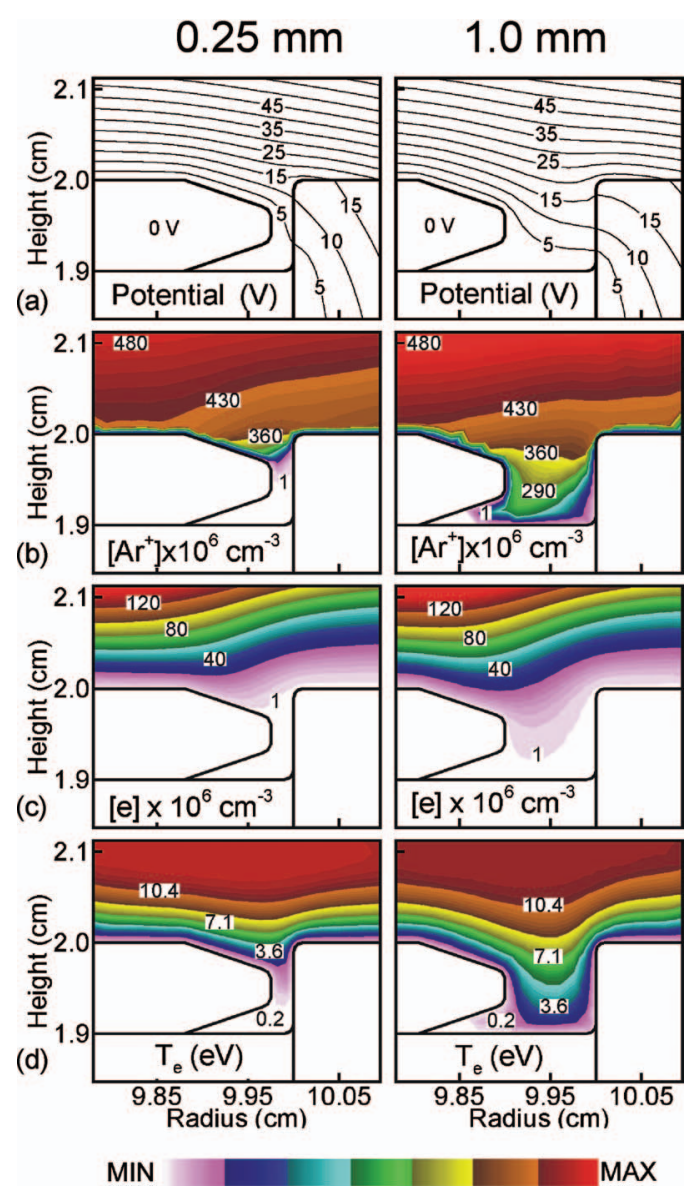

FIG. 7. (Color) Plasma properties averaged over the rf cycle for (left) a 0.25 $\mathrm{mm}$ gap and (right) a $1 \mathrm{~mm}$ gap for the base case conditions. (a) Plasma potential, (b) $\mathrm{Ar}^{+}$density, (c) electron density, and (d) electron temperature.

operating conditions $\left(\mathrm{Ar} / \mathrm{CF}_{4}\right.$ mixture at a gas pressure of 90 mTorr) the effective molecular mean free path is of the order of $0.6 \mathrm{~mm}$. Thus, for gaps with dimensions smaller than 0.5 $\mathrm{mm}$, the Knudsen number is near or greater than one, and the mean free path of a molecule is comparable to a length scale of the problem.

\section{REACTION MECHANISM}

The purpose of this investigation is to discuss the consequences of plasma penetration into the wafer-focus ring gap, as opposed to optimizing a particular plasma process for a specific application. As a result, a relatively simply reaction mechanism was chosen that captures the major effects we wish to address (e.g., radical generation and possible deposition in an electronegative gas mixture). To this end, simulations were performed for an $\mathrm{Ar} / \mathrm{CF}_{4}$ gas mixture using a subset of the reactions discussed in Ref. 8. The species included in the reduced reaction mechanism are $\operatorname{Ar}, \operatorname{Ar}(4 s)$, $\mathrm{Ar}^{+}, \mathrm{CF}_{4}, \mathrm{CF}_{3}, \mathrm{CF}_{2}, \mathrm{CF}, \mathrm{F}, \mathrm{CF}_{3}^{+}, \mathrm{CF}_{3}^{-}$, and $\mathrm{F}^{-}$. The subset of reactions from Ref. 8 we used is listed in Table I.

For demonstration purposes, a simple polymer depositing surface reaction mechanism was used. Although the actual deposition process can be considerably more complicated involving, for example, production of high mass neutrals ${ }^{9}$ and creation of activated sites, ${ }^{7}$ we used this simpler mechanism to expedite the study. The mechanism con- 

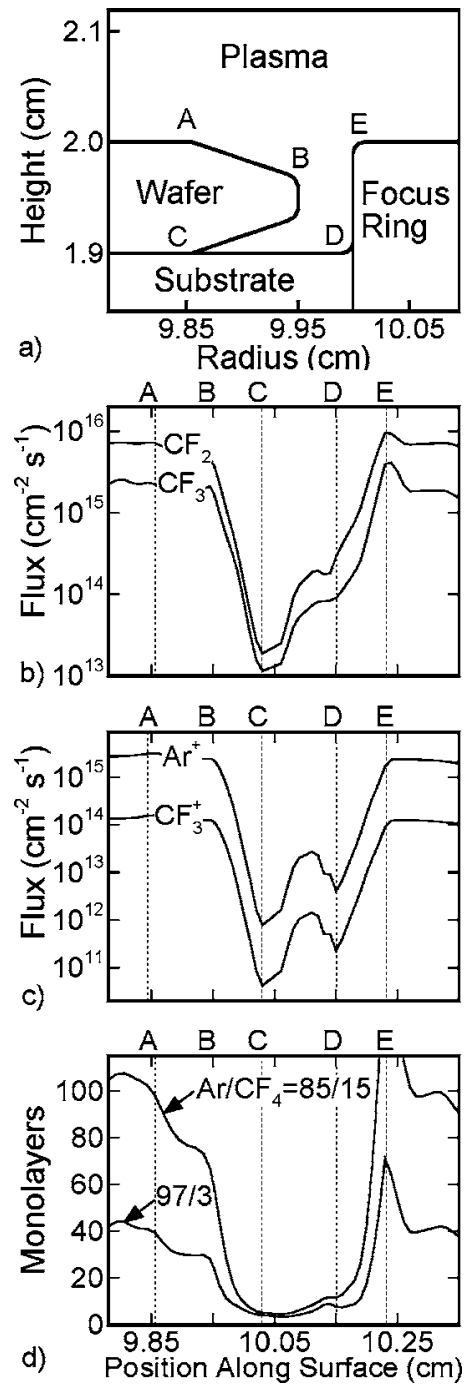

FIG. 8. Plasma properties along the surface of a $0.5 \mathrm{~mm}$ gap. (a) Schematic of the gap showing the reference locations cited below. (b) Fluxes of the polymerizing radicals $\mathrm{CF}_{2}$ and $\mathrm{CF}_{3}$, (c) fluxes of $\mathrm{Ar}^{+}$and $\mathrm{CF}_{3}^{+}$and (d) number of monolayers of polymer along the surface. Polymer layers are shown for $\mathrm{Ar} / \mathrm{CF}_{4}=97 / 3$ and 85/15 mixtures. Polymer deposition is disproportionately large in the gap due to the lack of ion sputtering.

sists of direct deposition of a fluorocarbon polymer by $\mathrm{CF}_{2}$ and $\mathrm{CF}_{3}$ radicals and the sputtering of the polymer by positive ions

$$
\begin{aligned}
& \mathrm{CF}_{2}+W \rightarrow P_{1}, p=0.1,0.2,0.3, \\
& \mathrm{CF}_{3}+W \rightarrow P_{1}, \quad p=0.05,0.1,0.2, \\
& \mathrm{CF}_{2}+P_{m} \rightarrow P_{m+1}, \quad p=0.4, \\
& \mathrm{CF}_{3}+P_{m} \rightarrow P_{m+1}, \quad p=0.3, \\
& M^{+}+P_{1} \rightarrow M+P_{1}, \quad p=0.8, \\
& M^{+}+P_{m} \rightarrow P_{m-1}+M+\mathrm{CF}_{2}, \quad p=0.2,
\end{aligned}
$$

where $W$ is a bare wall or surface site, $P_{m}$ is a polymer layer of thickness $m, M^{+}$represents any ion, $M$ is the ion's neutral counterpart, and $p$ is the probability for the process. (Multiple probabilities are for the focus ring, substrate and wafer,
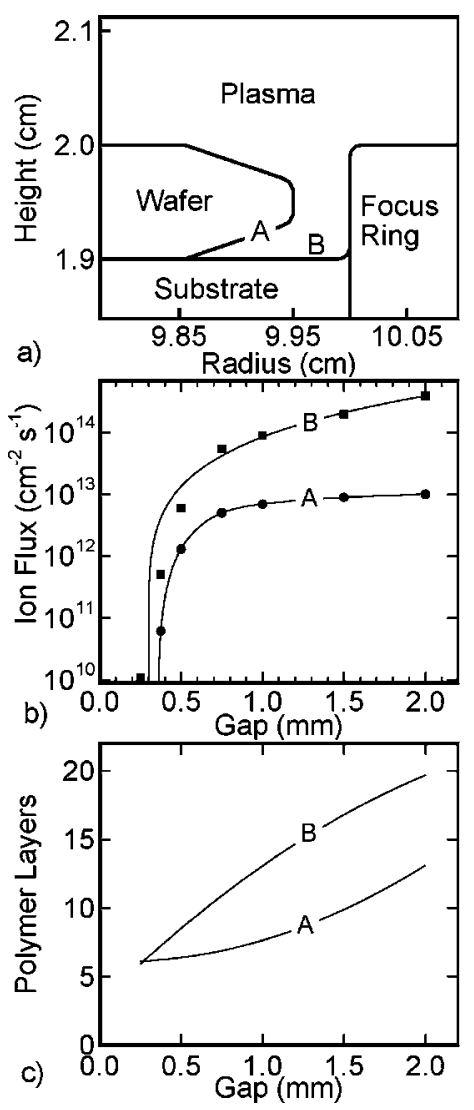

FIG. 9. Plasma properties within the gap as a function of gap size. (a) Schematic of the gap showing the reference locations A (below the bevel) and $\mathrm{B}$ (on the substrate with a view angle of the plasma). (b) Total ion fluxes and (c) polymer layers. Ion fluxes significantly decrease for gaps smaller than $0.75 \mathrm{~mm}$, approximately the sheath thickness.

respectively.) The passivation of the bare surface with polymer to the single mono-layer level is represented by Eqs. (9a) and (9b). The deposition of polymer on top of polymer to produce multiple layers is represented by Eq. (9c) and (9d). The sputtering of the sub-monolayer thickness of polymer to uncover bare surface sites is in Eq. (9c). The sputtering of polymer layers greater than one is represented by Eqs. (9e) and (9f). The probabilities in Eq. (9a)-(9f) are approximations based on previous, more detailed studies as described in Ref. 7.

\section{PLASMA AND RADICAL PENETRATION INTO THE WAFER-FOCUS RING GAP}

The plasma conditions used in this study are an $\mathrm{Ar} / \mathrm{CF}_{4}$ gas mixture at 90 mTorr in a capacitively coupled discharge with a $300 \mathrm{~V}$ amplitude applied to the substrate at $10 \mathrm{MHz}$. The base case gas mixture is $\mathrm{Ar} / \mathrm{CF}_{4}=97 / 3$. The substrate of the reactor, schematically shown in Fig. 1, is metal and powered. The focus ring is a dielectric $\left(\varepsilon / \varepsilon_{0}=4.0\right)$ with a negligible conductivity. The wafer has a conductivity of $0.1 \Omega^{-1} \mathrm{~cm}^{-1}$ with $\varepsilon / \varepsilon_{0}=8.0$. The unstructured mesh has multiple refinement zones to gradually resolve the small dimensions of the gap. One goal of this work is to resolve the entire problem in a single mesh as opposed to using a multimesh technique. As a consequence, the total computer time increases with increasing resolution of the mesh. Although 

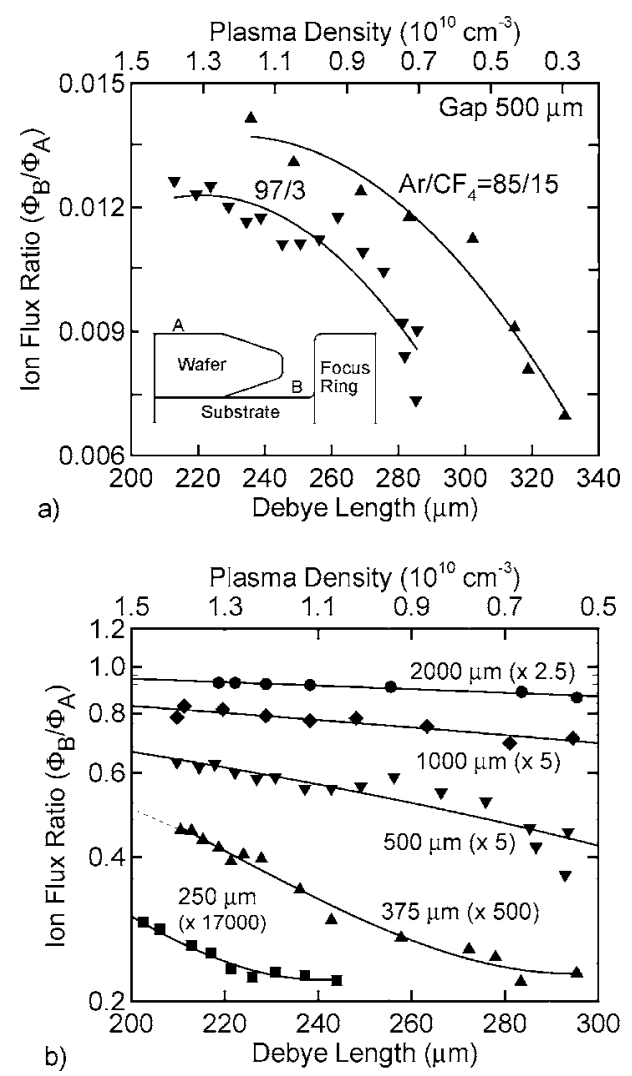

FIG. 10. The ratio of ion flux $\alpha$ arriving to the substrate with view angle to the plasma (point B) compared to the wafer (point A) as a function of the Debye length and plasma density. (a) $\alpha$ for a $0.5 \mathrm{~mm}$ gap for $\mathrm{Ar} / \mathrm{CF}_{4}$ $=85 / 15$ and $97 / 3$. (b) $\alpha$ for $A r / \mathrm{CF}_{4}=97 / 3$ for different gap sizes. $\alpha$ decreases with gap size and, therefore, has been rescaled to fit within a single decade for clarity. The inset in the top figure shows the positions of points A and $\mathrm{B}$. With gaps smaller than the Debye length, $\alpha$ is small indicating a rapid decrease of flux inside the gap. As the gap size becomes large compared to the Debye length, $\alpha$ approaches unity.

we believe the number of mesh points within the gap is sufficient to resolve the problem (confirmed by performing numerical experiments), resolving small structures will always be a tradeoff between the degree of resolution and computer time.

Cycle averaged plasma parameters are shown in Fig. 2 for the base case. Ion density and $T_{e}$ along the axis are shown in Fig. 3. The electron density has a peak value of 6 $\times 10^{9} \mathrm{~cm}^{-3}$ located near the edge of the wafer, with an on axis maximum of $2 \times 10^{9} \mathrm{~cm}^{-3}$. The negative ion density has an on axis maximum value of $1 \times 10^{10} \mathrm{~cm}^{-3}$, an electronegativity of about 5 . This electronegativity enables the negativeion balanced positive ion density to have a maximum value of $1.4 \times 10^{10} \mathrm{~cm}^{-3}$ with a radial uniformity of about $15 \%$. In spite of the large balance of argon in the mixture, the ratio of $\mathrm{Ar}^{+}$to $\mathrm{CF}_{3}^{+}$ions is only about 6 . Radical densities are dominated by $\mathrm{CF}_{2}$ with a maximum value of $1 \times 10^{12} \mathrm{~cm}^{-3}$.

The penetration of plasma into the wafer-focus ring gap depends on a number of factors, including charging of the adjacent structures, size of the gap compared to the sheath thickness, and physical configuration of the edge of the wafer. For example, the plasma potential, electron density, and $\mathrm{Ar}^{+}$density for gaps of 0.25 and $1 \mathrm{~mm}$ are shown in the vicinity of the gap at different times during the rf cycle in
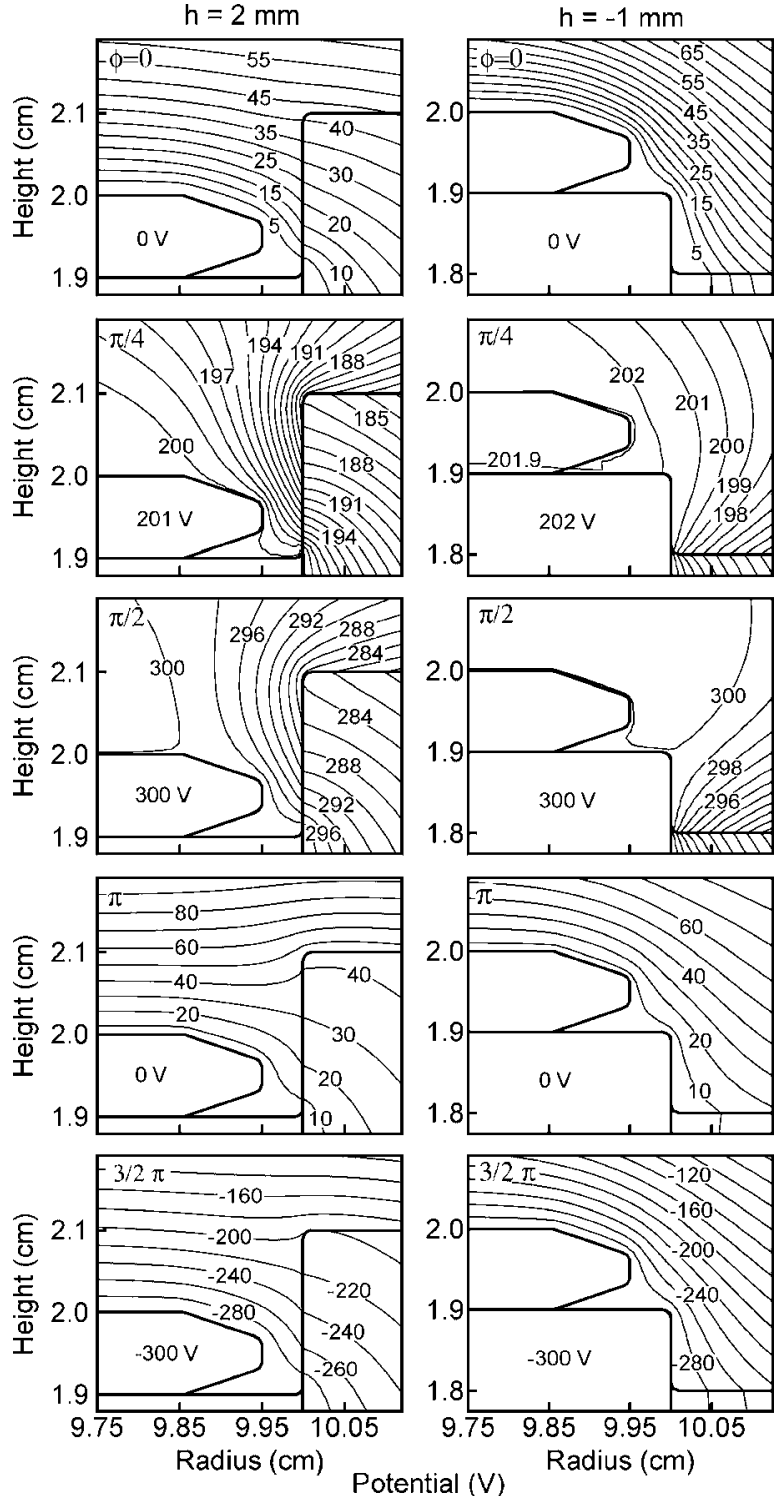

FIG. 11. Plasma potential (volts) at different phases during the rf cycle for (left) a high ring $(h=2 \mathrm{~mm})$ and (right) a low ring $(h=-1 \mathrm{~mm})$ for the base case conditions. The maximum voltage occurs at $\phi=\pi / 2$ and minimum voltage at $3 \pi / 2$.

Figs. 4-6. (The width of the gap is the horizontal distance between the vertical edge of the wafer and the interior sidewall of the focus ring.) Cycle average values of these quantities and for $T_{e}$ are in Fig. 7. The conductivity of the wafer is sufficiently high that there is little voltage drop across it (a few volts at most) though not so large that there is no charge accumulation on its surface. The dielectric focus ring does charge and discharge during the rf cycle.

The sheath width for these conditions is a few $\mathrm{mm}$, larger than the $0.25 \mathrm{~mm}$ gap but commensurate with the 1 mm gap. As such, the charged particle densities in and near the gap are typically in a non-neutral situation. The lack of strong ambipolar forces, combined with the large electric fields that accelerate charged particles into and out of the gap, allows there to be differential rates of loss between the electrons and positive ions. (No negative ions penetrate into the gap for these conditions.) At no time during the rf cycle is there significant charged particle penetration into the 0.25 


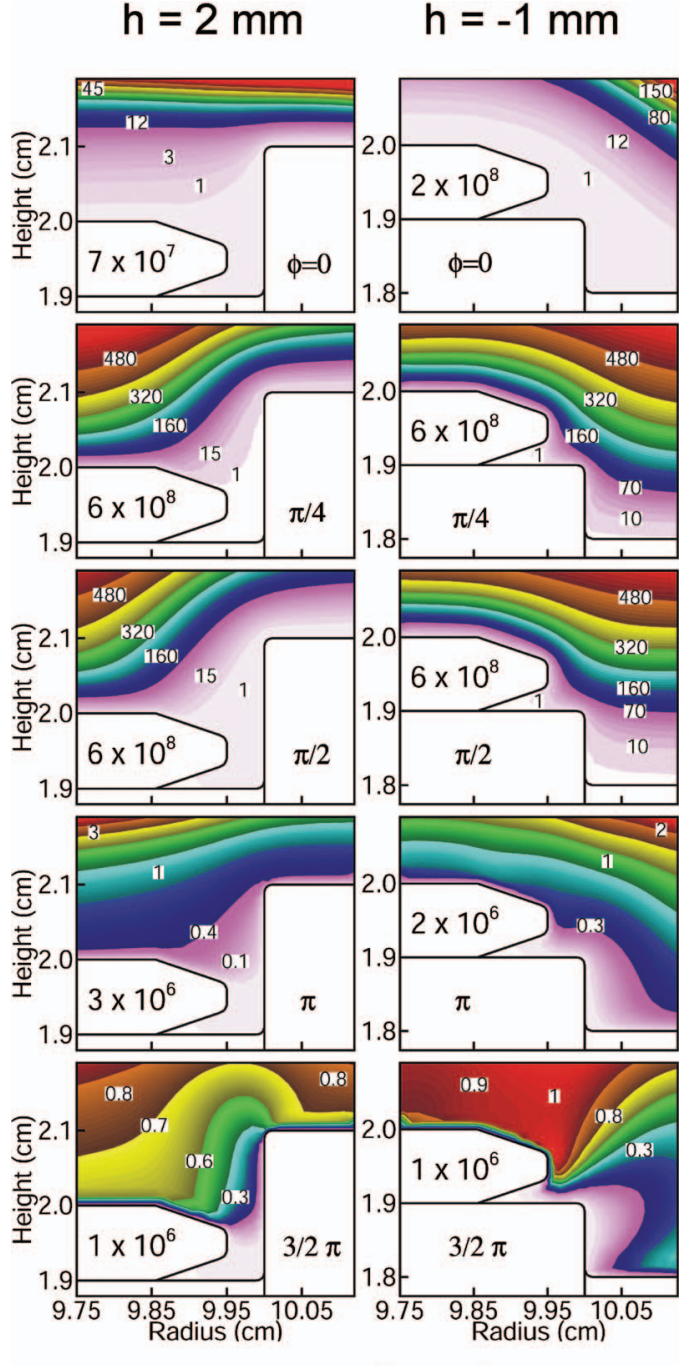

[e] $\times 10^{6} \mathrm{~cm}^{-3}$
MAX

FIG. 12. (Color) Electron density at different phases during the rf cycle for (left) a high ring $(h=2 \mathrm{~mm})$ and (right) a low ring $(h=-1 \mathrm{~mm})$ for the base case conditions. The maximum voltage occurs at $\phi=\pi / 2$ and minimum voltage at $3 \pi / 2$. Contour labels are in units of $10^{6} \mathrm{~cm}^{-3}$. Each frame is separately normalized to its maximum value. Electron penetration into the gap is diminished by increasing the height of the ring.

mm gap. Charging of surfaces create sufficiently large transverse fields to promote the collection of charge that might otherwise penetrate into the gap. For example, for the 0.25 $\mathrm{mm}$ gap even during the peak of the cathodic part of the cycle when ions should be accelerated into the gap, charging of the focus ring (and geometrical field warping) accelerate ions into the bevel of the wafer instead.

The electron density does not significantly exceed $10^{6} \mathrm{~cm}^{-3}$ in either the 0.25 or $1 \mathrm{~mm}$ gaps. The ion density is similarly excluded from the $0.25 \mathrm{~mm}$ gap but is able to penetrate to densities of $>10^{8} \mathrm{~cm}^{-3}$ in the $1 \mathrm{~mm}$ gap. The lower surface of the gap is a metal electrode and the wafer is sufficiently conductive that some, but not significant, charge accumulation occurs. The focus ring nearly continuously accumulates positive charge. It is only momentary spurts of

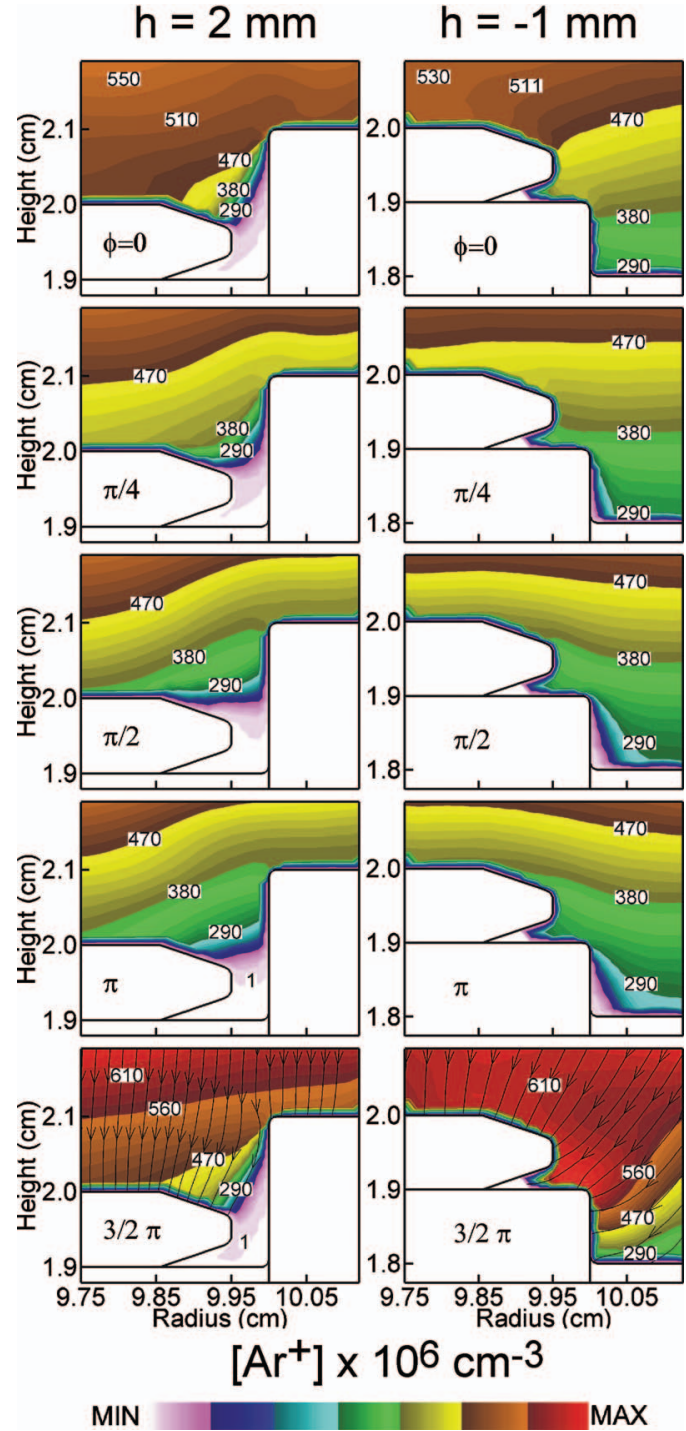

FIG. 13. (Color) $\mathrm{Ar}^{+}$density at different phases during the rf cycle for (left) a high ring $(h=2 \mathrm{~mm})$ and (right) a low ring $(h=-1 \mathrm{~mm})$ for the base case conditions. The maximum voltage occurs at $\phi=\pi / 2$ and minimum voltage at $3 \pi / 2$. Contour labels are in units of $10^{6} \mathrm{~cm}^{-3}$. Ion flux streamlines are shown in the last frame.

electrons into the gap during the anodic part of the cycle that allows a cycle averaged neutralization of the positive charge on the focus ring.

The electron temperature is obtained from a collisional heating, continuum model and, therefore, is at best an approximation in the large Knudsen number volume of the gaps. Having said that, the cycle averaged values of $T_{e}$ shown in Fig. 7 are additional indications of the disparity between these two gaps. Though the electron density is low in the $1 \mathrm{~mm}$ gap, $T_{e}$ exceeds $3-4 \mathrm{eV}$, indicating that energy transport into the gap is important whereas for the $0.25 \mathrm{~mm}$ gap, energy transport into the gap is not important.

The cycle averaged fluxes of ions and polymerizing radicals to the surfaces in and around the $0.5 \mathrm{~mm}$ gap are shown in Fig. 8. The number of layers of polymer on the surface after $20 \mathrm{~s}$ of processing is also shown for $\mathrm{Ar} / \mathrm{CF}_{4}=97 / 3$ and $85 / 15$ mixtures. The ion flux, dominated by $\mathrm{Ar}^{+}$, is 3 $\times 10^{15} \mathrm{~cm}^{-2} \mathrm{~s}^{-1}$ on the top surface of the wafer and focus 


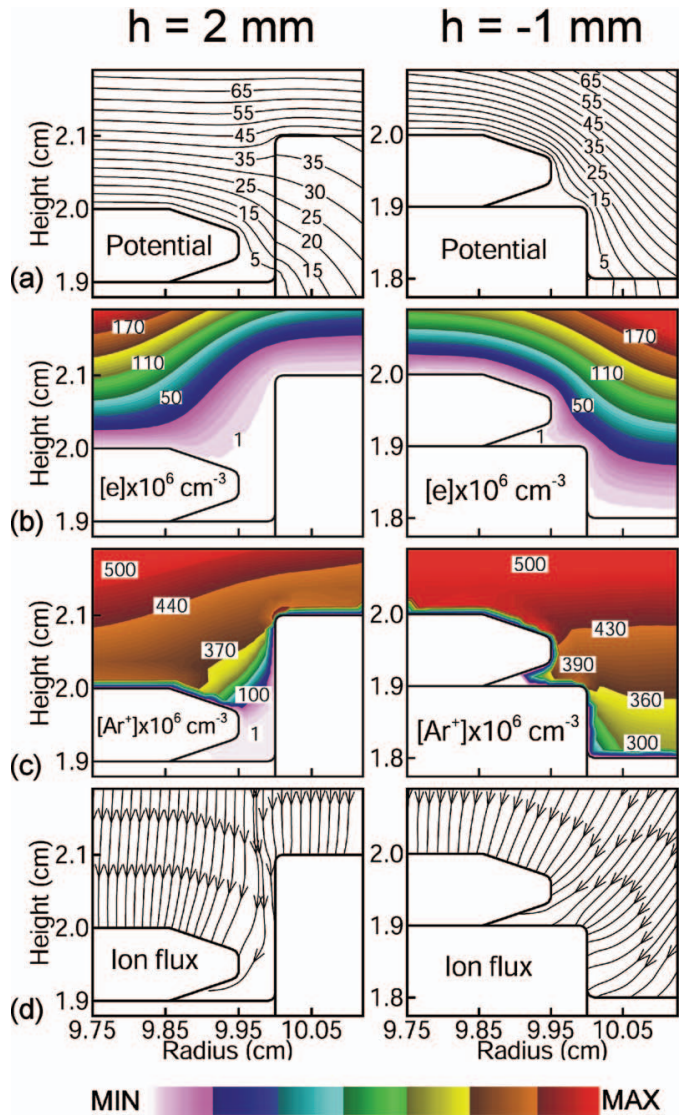

FIG. 14. (Color) Plasma properties averaged for the rf cycle for (left) a high ring $(h=2 \mathrm{~mm})$ and (right) a low ring $(h=-1 \mathrm{~mm})$ the base case conditions (a) Plasma potential (b) electron density, (c) $\mathrm{Ar}^{+}$density, and (d) ion flux streamlines. Penetration of plasma under the wafer is significant for the low ring case.

ring. The flux diminishes to $10^{12} \mathrm{~cm}^{-2} \mathrm{~s}^{-1}$ on the underside of the bevel and $2 \times 10^{13} \mathrm{~cm}^{-2} \mathrm{~s}^{-1}$ on the substrate having a view angle to the plasma. The local maximum in ion flux on the substrate where there is a view angle to the plasma is an indication of the directed ion momentum through the gap. The ion flux to the underside of the bevel is likely larger than in reality due to the continuum approximation used here which places greater emphasis on diffusive transport compared to a ballistic kinetic transport.

The polymerizing radical flux has a similar decrease as do the ions in penetrating into the gap. The difference in flux from the top of the wafer, $10^{16} \mathrm{~cm}^{-2} \mathrm{~s}^{-1}$, to the substrate with a view angle to the plasma is a factor of $10^{3}$. Note that the neutral radical flux, being dominated by diffusive transport and lacking the directed component, does not have as large a local maximum on the substrate under the gap as do the ions.

The number of layers of polymer on the surface in and around the gap, shown in Fig. 8(d), decreases from about 40 for the $\mathrm{Ar} / \mathrm{CF}_{4}=97 / 3$ mixture and 110 for the $\mathrm{Ar} / \mathrm{CF}_{4}$ $=85 / 15$ mixture, to about 5 on the substrate under the gap. The decrease is only a factor of 8 in the leaner mixture in spite of the polymerizing flux decreasing by a factor of 100 . The greater than expected deposition in the gap results from the even larger decrease in the ion flux which would otherwise sputter the polymer. In fact, the deposition on the underside of the bevel is nearly 10 times more efficient than on
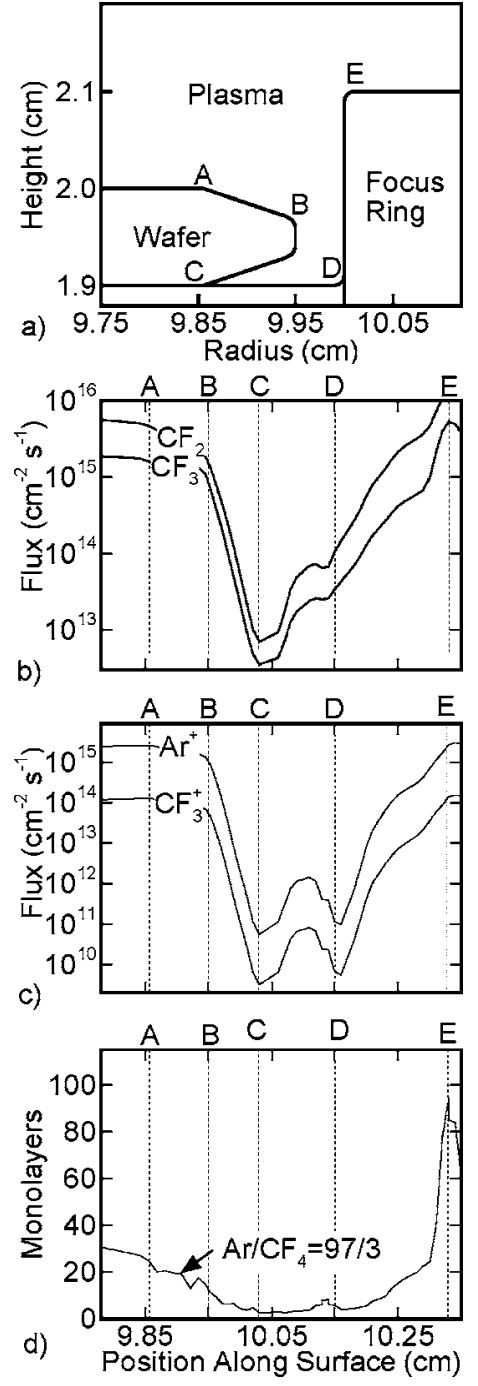

FIG. 15. Plasma properties along the surface of a $0.5 \mathrm{~mm}$ gap and $2 \mathrm{~mm}$ focus ring height. (a) Schematic of the gap showing the reference locations, (b) fluxes of the polymerizing radicals $\mathrm{CF}_{2}$ and $\mathrm{CF}_{3}$, (c) fluxes of $\mathrm{Ar}^{+}$and $\mathrm{CF}_{3}^{+}$and (d) number of monolayers of polymer along the surface. Polymer deposition is disproportionately large in the gap due to the lack of ion sputtering.

the substrate under the gap due to the lack of ion sputtering. This propensity for deposition on surfaces without a direct line of site to the plasma (and, therefore, lacking energetic ion sputtering) will likely also correlate with the formation of particles.

The ion flux and polymer layers under the bevel of the wafer (point A) and on the substrate with the view angled to the plasma (point B) are shown in Fig. 9 as a function of width of the gap. The flux to the bevel is dominantly diffusive (and, therefore, likely overestimated here) whereas that to the substrate is dominantly directed. Between gap widths of 0.7 and $2 \mathrm{~mm}$, the flux to the bevel is relatively constant whereas the flux to the substrate increases by a factor of 4 to 5 The plasma density, a few $\mathrm{mm}$ above point $\mathrm{B}$, does not appreciably change. The increase in flux is largely a consequence of the decrease in lateral electric fields due to the charging of surfaces as the gap widens. (See, for example, Fig. 4.) As the gap decreases below $0.7 \mathrm{~mm}$, approximately the sheath width, there is a rapid decrease in ion flux. As the 


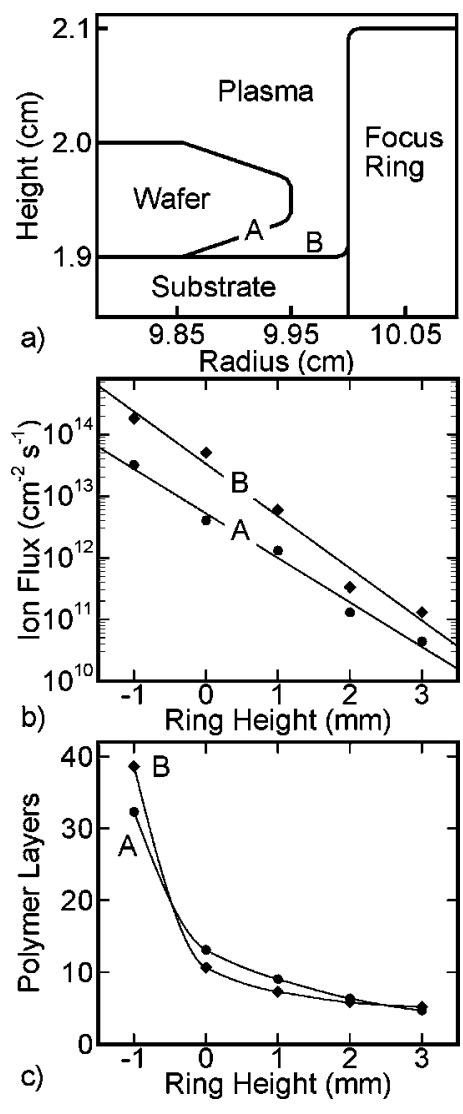

FIG. 16. Plasma properties in the vicinity of the wafer edge as a function of height of the focus ring. (a) Schematic of the gap showing the reference locations A (below the bevel) and B (on the substrate with a view angle of the plasma), (b) total ion fluxes and (c) polymer layers. Polymer deposition significantly decreases for heights above $1 \mathrm{~mm}$.

width of the gap decreases significantly below the sheath thickness, the ability for the plasma to conform to its interior surfaces diminishes. Ions will more likely be directed into the side walls of the dielectric and edges of the bevel at the top of the gap than penetrate into the gap.

The layers of polymer also decrease with decreasing gap size, though at best that decrease is linear as opposed to the exponential decrease in ion flux. Although the polymer thickness under the bevel (point A) is only $2 / 3$ of that directly under the gap (point B) when the gap is large, the rate of decrease in thickness is less as the gap gets smaller. For small gaps $(0.25 \mathrm{~mm})$ the thickness in polymer in both locations is nearly the same, though polymer deposition at point A is usually smaller than at point $\mathrm{B}$. This reflects the small ion fluxes to either location that remove the polymer by sputtering.

Ion assisted deposition usually results from low energy ion bombardment activating surface sites and whereas ion sputtering of polymer occurs from high energy ion bombardment. ${ }^{7}$ As such, including ion assisted deposition would have required resolving the ion energy distributions in the gap, a capability not presently available. We believe that had we included these processes, the effects we speak about would have been magnified. The ions incident on, for example, surfaces under the bevel would have been of lower energy than those sites having a view angle of the plasma. The propensity for polymer deposition on those surfaces would, therefore, have been larger.

The ratio $\alpha$ of ion flux arriving on the substrate with view angle to the plasma (point $\mathrm{B}$ ) compared to the ion flux to the top of the wafer (point A) is shown in Fig. 10(a) for a $0.05 \mathrm{~mm}$ gap as a function of the Debye length and plasma density. $\alpha$ is shown in Fig. 10(b) for different gap sizes as a function of Debye length and plasma density. With gaps smaller than the Debye length (that is typically a measure of the sheath thickness) $\alpha$ is small. This is a situation where the sheath largely sits above the gap and is unable to mold to the internal boundaries of the gap. With an increase of plasma density or decrease in the Debye length, $\alpha$ increases for any gap size. The smaller the gap size the larger the fractional increase. This indicates that the sheath is better able to mold along the interior of the gap thereby enabling a greater penetration of the plasma into the gap. For large gaps, such as the $2 \mathrm{~mm}$ gap in Fig. 10(b), $\alpha$ is nearly independent of Debye length and near unity for the range investigated (200 $-300 \mu \mathrm{m})$ as the plasma is able to penetrate to the substrate in all cases. For smaller gaps, higher plasma densities (smaller Debye lengths) are needed for the plasma to penetrate to the substrate. These results imply that for high plasma density reactors $\left(n_{e}>10^{11} \mathrm{~cm}^{-3}\right)$, the plasma will likely penetrate into gaps of $<100 \mu \mathrm{m}$.

Another design variable is the height of the focus ring, $h$, relative to the substrate. (Positive values of $h$ denote focus rings whose top surface is above the substrate; negative values of $h$ are for focus rings below the substrate.) The electric potential, electron density, and $\mathrm{Ar}^{+}$density at different time during the rf phase are shown for high $(h=2 \mathrm{~mm})$ and low $(h=-1 \mathrm{~mm}$ ) ring heights for a $0.5 \mathrm{~mm}$ gap in Figs. 11-13. The cycle average values of potential, electron density, $\mathrm{Ar}^{+}$, and ion flux are shown in Fig. 14. When the focus ring is high, the underside of the bevel is even more tightly shielded. When the focus ring is low, the underside of the bevel is exposed, as is the substrate.

At the peak of the cathodic part of the cycle (phase $3 \pi / 2$ ), electrons are basically absent from under the bevel for either the high or low focus ring. At the peak of the anodic part of the cycle (phase $\pi / 2$ ), electron penetration under the bevel should be at a maximum. Nevertheless, there is negligible penetration of plasma under the bevel with the high focus ring, whereas with the low focus ring, electrons penetrate many $\mathrm{mm}$ under the bevel. In both cases, the top surface of the dielectric charges negatively. The fact that this negative charge is above the bevel in the former case and below the bevel in the latter case, has important implications with respect to the ion flux.

Whereas there is significant penetration of ions into a 0.5 $\mathrm{mm}$ gap for the base case, there is nominal penetration for a ring height of $2 \mathrm{~mm}$ at any time during the rf cycle. Even at the peak of the cathodic part of the cycle, ion trajectories curve into the top surface of the bevel. On the other hand, the ion flux is continuously directed to the underside of the bevel throughout the rf cycle for negative heights of the focus ring. Here, the transient negative charging of the dielectric is important. For high focus rings, the negative charging pulls 
ions away from vertices of the wafer and the bevel. For low focus rings, the negative charging pulls ions down into the vicinity of the vertices and bevel of the wafer.

The streamlines of ion fluxes averaged over the rf cycle shown in Fig. 14(d). In the case of the high focus ring, there are only errant ion trajectories that penetrate through the gap. As such, there is also little, if any, focusing of the ion flux to the edge of the bevel of the wafer. There is some ion focusing to the edge of the dielectric focus ring. In the case of the low focus ring, ion trajectories readily reach and focus onto the edge of the wafer and below the bevel. Ion focusing is also intense onto the edge the substrate.

The cycle averaged fluxes of ions and polymerizing radicals to the surfaces in and around the $0.5 \mathrm{~mm}$ gap with $2 \mathrm{~mm}$ high focus ring are shown in Fig. 15. The number of layers of polymer on the surface after $20 \mathrm{~s}$ of processing is also shown in Fig. 15(d). The ion flux, dominated by $\mathrm{Ar}^{+}$, is 3 $\times 10^{15} \mathrm{~cm}^{-2} \mathrm{~s}^{-1}$ on the top surface of the wafer and focus ring. The ion flux diminishes to $10^{10} \mathrm{~cm}^{-2} \mathrm{~s}^{-1}$ on the underside of the bevel and $1 \times 10^{12} \mathrm{~cm}^{-2} \mathrm{~s}^{-1}$ on the substrate having a view angle to the plasma. In this case, the ion flux decreases by a factor of $10^{5}$ whereas for a $1 \mathrm{~mm}$ high focus ring (see Fig. 8) the decrease is a factor of $10^{3}$, The polymerizing radical flux has a similar decrease as the ions in penetrating into the gap. The difference in flux from the top of the wafer, $10^{16} \mathrm{~cm}^{-2} \mathrm{~s}^{-1}$, to the substrate with a view angle to the plasma is a factor of $10^{4}$, also a larger decrease than for the lower focus ring height. The number of layers of polymer on surface in and around the gap, shown in Fig. 15(d), does not decrease with the focus ring height as rapidly as the ion flux.

The ion flux and layers of polymer on the substrate and on the wafer below the bevel are shown in Fig. 16 as a function of height of the focus ring. The ion flux and polymer deposition both decrease with increasing ring height. Rings greater in height than the wafer ( $1 \mathrm{~mm}$ in this case) are effective in reducing the amount of polymer deposited below the bevel.

\section{CONCLUDING REMARKS}

In this paper, penetration of plasma into the wafer-focus ring gap of a capacitively coupled discharge was computationally investigated. It was shown that plasma penetration depends on the size of the gap relative to sheath thickness or the Debye length. For the test conditions $\left(\mathrm{Ar} / \mathrm{CF}_{4}, 90 \mathrm{mTorr}\right.$, $300 \mathrm{~V},\left[M^{+}\right]=10^{10} \mathrm{~cm}^{-3}$ ) significant penetration occurs for gaps $>0.5 \mathrm{~mm}$. For high plasma densities more penetration occurs into gaps of similar sizes due to smaller sheaths and more conformal molding of the sheath into the interior of the gap. Polymerization inside the gap relative to, for example, on top of the wafer is magnified by a disproportionate reduction in ion sputtering.

\section{ACKNOWLEDGMENTS}

This work was supported by the Semiconductor Research Corporation and the National Science Foundation (CTS-0520368).

${ }^{1}$ B. J. Jeon, H. Y. Chang, J. K. Song, and C. W. Jeon, Plasma Sources Sci. Technol. 11, 520 (2002).

${ }^{2}$ Handbook of Plasma Processing Technology, edited by S. M. Rossnagel, J. J. Cuomo, and W. D. Westwood (Noyes, Park Ridge, N.J., 1990).

${ }^{3}$ U. Czarnetzki, G. A. Hebner, D. Luggenholscher, H. F. Dobele, and M. E. Riley, IEEE Trans. Plasma Sci. 27, 70 (1999).

${ }^{4}$ D. Kim and D. J. Economou, IEEE Trans. Plasma Sci. 30, 2048 (2002).

${ }^{5}$ D. Kim, D. J. Economou, J. R. Woodworth, P. A. Miller, R. J. Shul, B. P. Aragon, T. W. Hamilton, and C. G. Willison, IEEE Trans. Plasma Sci. 31, 691 (2003).

${ }^{6}$ M. J. Kushner, J. Phys. D 38, 1633 (2005).

${ }^{7}$ D. Zhang and M. J. Kushner, J. Appl. Phys. 87, 1060 (2000).

${ }^{8}$ A. V. Vasenkov, X. Li, G. S. Oehrlein, and M. J. Kushner, J. Vac. Sci. Technol. A 22, 511 (2004).

${ }^{9}$ G. Cunge and J. P. Booth, J. Appl. Phys. 85, 3952 (1999).

${ }^{10}$ K. Tachibana, Phys. Rev. A 34, 1007 (1986).

${ }^{11}$ D. Rapp and P. Englander-Golden, J. Chem. Phys. 43, 1464 (1965).

${ }^{12}$ R. H. McFarland and J. D. Kinney, Phys. Rev. 137, A1058 (1965).

${ }^{13}$ I. P. Zapesochnyi, Y. N. Semenyuk, A. I. Dashchenko, A. E. Imre, and A. I. Zapesochnyi, JETP Lett. 8, 260 (1984).

${ }^{14}$ L. Vriens, Phys. Lett. 8, 260 (1964).

${ }^{15}$ R. A. Bonham, Jpn. J. Appl. Phys., Part 1 33, 4157 (1994).

${ }^{16}$ V. Tarnovsky, P. Kurunczi, D. Rogozhnikov, and K. Becker, Int. J. Mass Spectrom. Ion Process. 128, 181 (1993).

${ }^{17}$ M. Hayashi and T. Nimura, J. Appl. Phys. 54, 4879 (1983).

${ }^{18}$ A. N. Klucharev and V. Vujnovic, Phys. Rep. 185, 55 (1990).

${ }^{19}$ I. C. Plumb and K. R. Ryan, Plasma Chem. Plasma Process. 9, 409 (1989).

${ }^{20}$ C. Tsai, S. M. Belanger, J. T. Kim, J. R. Lord, and D. L. McFadden, J. Phys. Chem. 93, 1916 (1989).

${ }^{21}$ D. R. Burgess, M. R. Zachariah, W. Tsang, and P. R. Westmoreland, Prog. Energy Combust. Sci. 21, 453 (1995).

${ }^{22}$ N. I. Butkovskaya, M. N. Larichev, I. O. Leipunskii, I. I. Morozov, and V. L. Talroze, Kinet. Katal. 21, 263 (1980).

${ }^{23}$ J. E. Velazco, J. H. Kolts, and D. W. Setser, J. Chem. Phys. 65, 3468 (1976).

${ }^{24}$ H. W. Ellis, R. Y. Pai, E. W. McDaniel, E. A. Mason, and L. A. Viehland At. Data Nucl. Data Tables 17, 177 (1976).

${ }^{25}$ G. I. Font, W. L. Morgan, and G. Mennenga, J. Appl. Phys. 91, 3530 (2002).

${ }^{26}$ G. K. Vinogradov, P. I. Verzorov, L. S. Polak, and K. I. Slovetsky, Vacuum 32, 529 (1982).

${ }^{27}$ P. Ho, J. E. Johannes, R. J. Buss, and E. Meeks, J. Vac. Sci. Technol. A 19, 2344 (2001).

${ }^{28}$ E. R. Fisher, M. E. Weber, and P. B. Armentrout, J. Chem. Phys. 92, 2296 (1990).

${ }^{29}$ M. A. Biondi, Principles of Laser Plasmas, edited by G. Bekefi (Wiley, New York, 1976).

${ }^{30}$ R. E. Olson, J. R. Peterson, and J. Moseley, J. Chem. Phys. 53, 3391 (1970). 